\title{
CORRIGENDUM
}

\section{FRA16D common chromosomal fragile site oxido-reductase (FOR/ WWOX) protects against the effects of ionizing radiation in Drosophila}

\author{
LV O'Keefe, Y Liu, A Perkins, S Dayan, R Saint and RI Richards
}

Oncogene (2006) 25, 7662. doi:10.1038/sj.onc.1210119

Correction to: Oncogene (2005) 24, 6590-6596. doi:10.1038/sj.onc.1208806; published online 20 June 2005

Subsequent to our publication in Oncogene, several experiments have now led us to believe that the radiation sensitivity we observed in the $D m W W O X^{1}$ mutant strain was not due to the targeted mutation introduced in the $D m W W O X$ gene, but was a consequence of perturbations elsewhere in the genome.

These experiments were carried out with an additional mutant strain that was generated by insertion of the PiggyBac transposable element into the second intron of the DmWWOX gene. This independent allele of DmWWOX ( $\left.p B a c\{W H\} C G 7221^{f 04545}\right)$ was also sensitive to ionizing radiation, consistent with our reported findings. However, radiation sensitivity was not observed in animals that were trans-heterozygous for these two alleles. We therefore carried out four rounds of back-crossing of flies carrying either the homologous recombination targeted $\operatorname{Dm} W W O X^{I}$ allele or the PiggyBac insertion allele to wild-type flies and found that radiation sensitivity was significantly reduced in either of the resultant homozygous mutant lines. Thus, mutagenesis of the DmWWOX gene by the completely independent methods of homologous recombination targeting and PiggyBac mediated insertion induced additional alterations in the genome that conferred sensitivity to ionizing radiation. The crosses we undertook to reintroduce either the Drosophila or human WWOX gene for rescue of the radiation sensitivity presumably served to reduce the effect of the background mutation(s) responsible for this phenotype by outcrossing chromosomes that had undergone HR procedures and replacing them with wild-type chromosomes.

That these background mutations arise and can confound interpretation of phenotype data in Drosophila mutation experiments has implications for the general use of these mutagenesis approaches and will be the subject of more detailed analysis elsewhere. 